\title{
Sound velocity profiles in fluids for process monitoring
}

\author{
Lenz, Michael; Kühnicke, Elfgard \\ Technische Universität Dresden \\ Institut für Festkörperelektronik \\ D-01062 Dresden
}

\begin{abstract}
In conventional methods the sound velocity is determined by means of the back wall echo or a reflector at a known position. This paper presents a novel approach for combined measurement of sound velocities and distances in fluids with scattering particles. The basic idea is that the echo becomes strongest when the scattering particle is located within the maximum of the sound field, and thus the position of that maximum along the measuring depth is related to the according time of flight.

The paper gives proof of concept by measurements in fluids covering a wide range of sound velocities with an ultrasonic transducer equipped with a lens. In a detailed measurement using water at different temperatures, an uncertainty in velocity determination of less than $0.1 \%$ was achieved. Further experiments and simulations with an annular array demonstrate that the focus position can be varied without moving the transducer and thus sound velocity profiles can be measured.
\end{abstract}

\section{Introduction}

Methods for non-invasive determination of sound velocity in fluids are of great interest in process control, environmental measurement technology and medicine, because sound velocity can be used as an indicator of temperature, concentration or mass density [14].

By now, the majority of methods assume the propagation path length to be known in advance of sound velocity determination [5]. The novel method introduced in this paper does not use a reflector at a specific position, but rather makes use of the reflected wave from the scattering particles contained in the fluid. Neither the propagation path length nor the sound velocity are known in advance, so that both measurands need to be determined in combination.

The basic idea about the novel method is that the information on focus position suffices to determine the sound velocity of the fluid and that in turn, sufficient information on the transducer's focus position is contained in the echo signals. Proof of concept for these statements is given by theory and experiment with a focusing transducer and an annular array.

Since annular arrays allow tuning the focus position, it is also possible to resolve the sound velocity spatially and thus achieve sound velocity profiles. By that way, spatially resolved information about sound velocity from inside the fluid can be gathered with hardly any effect on the technological process.

\section{Discussion of feasibility on the basis of a circular piston transducer}

In general, the sound field of an ultrasonic transducer depends on both the transducer parameters (geometry and elastic material parameters) and the sound velocity of the propagation medium. Instead of using solely the propagation time, a second measurand - the focus position - is used, here. The focus position is usually measured by using a point reflector, where the measurement exploits that the maximum of the echo signal is achieved when the point reflector is located in the focus. The $z$-coordinate $F o k_{\text {Med }}$ of the focus position is given by the sound velocity $c$ and sound propagation time $t$ by:

$$
F_{0} k_{\text {Med }}=c_{\text {Med }} \cdot t
$$

The relation between focus position and sound velocity for a circular piston transducer can be expressed analytically and is used to clarify the method, here. As can be derived with the Rayleigh integral, the focus position Fok of a circular piston transducer equals

$$
\text { Fok }=\frac{d^{2}}{4 \lambda}=\frac{d^{2}}{4 c} \cdot f
$$

where $d$ is the disc diameter, $\square-$ the wavelength in the propagation medium and $f$ - the centre frequency of the transducer [6-8]. The focus position in the propagation medium Fok $_{\text {Med }}$ results from the focus position in a calibration medium $F o k_{\text {Cal }}$ by the following way:

$$
\frac{F_{o k_{\mathrm{Cal}}}}{F o k_{\mathrm{Med}}}=\frac{c_{\mathrm{Med}}}{c_{\mathrm{Cal}}}
$$


For a piston transducer, the focus distance is inversely proportional to the sound velocity of the propagation medium. Therefore, by using equations (1) and (3), both the focus distance and the sound velocity in a medium with unknown sound velocity can be determined in combination by

$$
\begin{array}{r}
F o k_{\text {Med }}^{2}=F o k_{\mathrm{Cal}} \cdot c_{\mathrm{Cal}} \cdot t \\
c_{\text {Med }}^{2}=\frac{F o k_{\mathrm{Cal}} \cdot c_{\mathrm{Cal}}}{t}
\end{array}
$$

without any further previous knowledge being required about the fluid. Unfortunately, a circular piston transducer is not suitable for measuring sound velocities reliably at high resolution. Since focusing is not strong enough, the time of flight to the sound field maximum cannot be detected safely. Therefore focusing transducers were used in the measurements.

\section{Sound velocity measurements using a focusing ultrasonic transducer equipped with a lens}

Measurements in different fluids covering a wide range of sound velocities

A series of measurements were carried out to proof the underlying concept. A strongly focusing ultrasonic transducer equipped with a lens was submerged into water and GalnSn (a liquid ternary metal alloy consisting of gallium, indium and tin), and the echo signal amplitudes (averaged over a multitude of measurements) were determined.

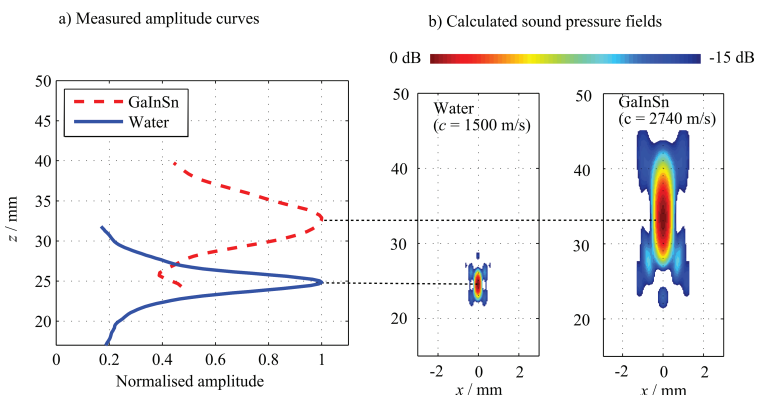

Figure 1 Comparison between averaged echo signal amplitude and focus position shows that the strongest echo signal is generated at the focus position.

Except for the metallic melt, which always contained enough natural impurities serving as scattering particles, all fluids were seeded with polyamid-12 powder with a particle size between 25 and $30 \mu \mathrm{m}$. As Figure 1 shows, the focus positions for different media are clearly distinguishable, and good agreement can be stated between the measured echo signal amplitudes shown in the leftmost panel and the simulated sound fields depicted in central and the rightmost panel. (See [9-11] for an explanation of the simulation algorithms.)

Note that - unlike with the circular piston transducer - the focus distance Fok increases with sound velocity, here. The reason is that the refractive power of the lens decreases the closer the sound velocity $c$ of the fluid approaches the sound velocity $C_{\text {Lens }}=5930 \mathrm{~m} / \mathrm{s}$ of the ultrasonic

\begin{tabular}{|c|c|c|}
\hline Fluid name & $\mathrm{T} /{ }^{\circ} \mathrm{C}$ & c / (m/s) \\
\hline ethanol 95\% & 50 & 1116 \\
\hline ethanol 95\% & 36 & 1169 \\
\hline ethanol 95\% & 20.7 & 1219 \\
\hline ethanol $70 \%$ & 23.9 & 1377 \\
\hline tap water & 6 & 1431 \\
\hline ethanol 50\% & 25.8 & 1499 \\
\hline tap water & 30 & 1509 \\
\hline tap water & 60 & 1551 \\
\hline glycerine $39 \%$ & 23.8 & 1685 \\
\hline glycerine $59 \%$ & 23.8 & 1785 \\
\hline glycerine $85 \%$ & 23.8 & 1879 \\
\hline GalnSn & 20 & 2740 \\
\hline \multicolumn{3}{|c|}{$\begin{array}{l}\text { Table } 1 \text { Fluids used in the } \\
\text { measurements. }\end{array}$} \\
\hline
\end{tabular}
lens.

Measurements of the same type were done in media with known sound velocities ranging between $1116 \mathrm{~m} / \mathrm{s}$ and $2740 \mathrm{~m} / \mathrm{s}$ (Table 1). The sound velocity values for the different media were taken from [12-17], where some values had to be interpolated with respect to temperature and concentration.

The corresponding measurements are depicted in Figure 2 and are compared to the sound field simulations. In the simulations the sound fields for a set of sound velocities were calculated, and the focus position was determined for each sound velocity. By converting the distances into the according times of flight, easier comparison between measurements and simulations was provided. Since the curve in Figure 2 is a monotonous function, the results are unambiguous. Thus, it can be used as a calibration curve for measurements in fluids with unknown sound velocity. In a measurement, the time of flight $t_{\mathrm{SFM}}$ to the focus is measured, and the sound velocity is simply read off of the curve [19-21]. 
Measurements in water of different temperatures, providing high resolution

Comparable measurements with fine resolution of the sound velocity were carried out in water of different temperatures. Temperature was varied between $6^{\circ} \mathrm{C}$ and $70^{\circ} \mathrm{C}$. By using a thermostat, temperature was kept within a range of $\pm 0.1^{\circ} \mathrm{C}$ around the nominal value.

The temperatures were converted into the sound velocity by using the $5^{\text {th }}$ order polynomial published in [1]. The corresponding curve is depicted in Figure 3.

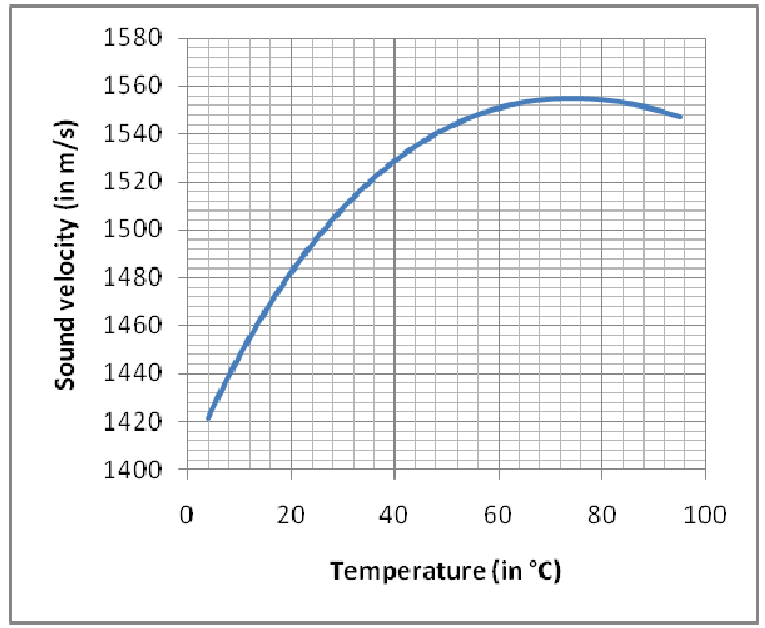

Figure 3 Sound velocity of water as a function of temperature.

As can be seen in the uppermost panel of Figure 4, the measured amplitude curves for the different temperatures are clearly distinguishable. The curve in the plot on the bottom can be seen as a highly resolved excerpt of the calibration function (Figure 2). It is linear, here, because its domain of definition is quite small.

The difference between the measurements and the fit curve equals $\sigma=1.4 \mathrm{~m} / \mathrm{s}$, which corresponds to a relative uncertainty of $\sigma / c \approx 0.1 \%$, thereby allowing precise measurements.

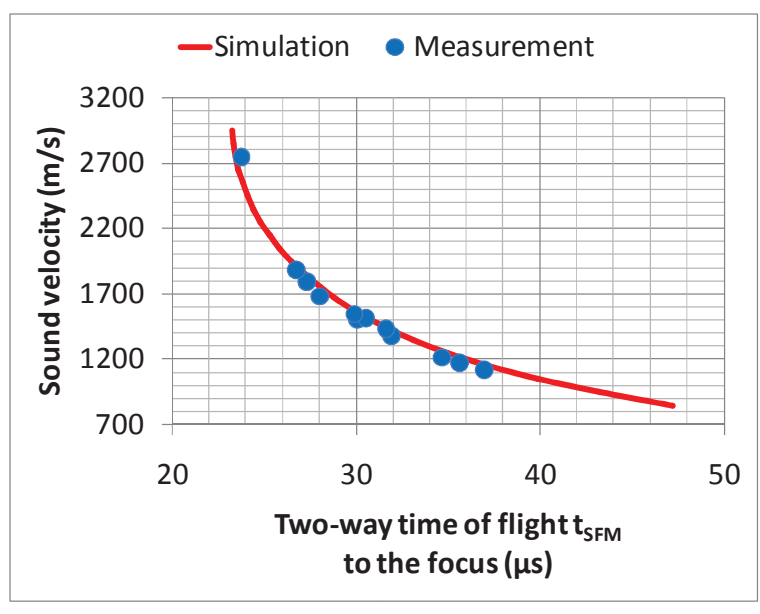

Figure 2 Sound velocity as a function of the two-way time of flight to the focus
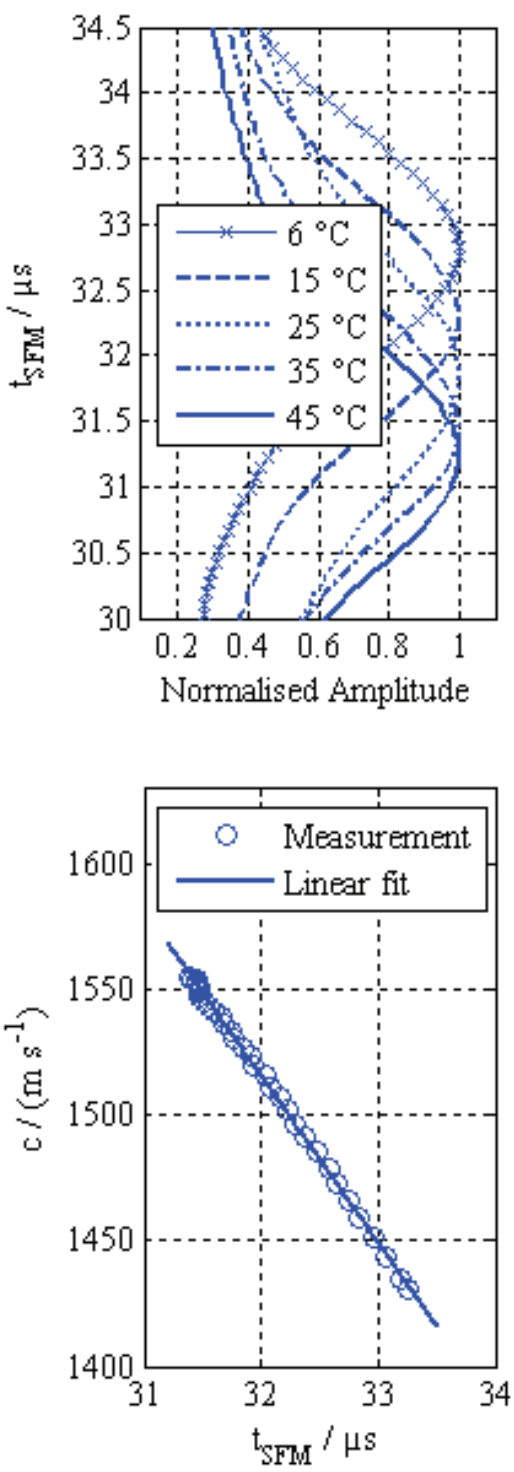

Figure 4 Measurements in water with fine adjustment of the sound velocity by temperature. 
Steps toward measuring sound velocity profiles using an ultrasonic annular array

Change of the focus position with an annular array

Measurements comprising synthetic focusing of an annular array were done in water with the sound velocity $c=1500 \mathrm{~m} / \mathrm{s}$ and a signal centre frequency of $9 \mathrm{MHz}$. The intended focus positions were 32,40 , and $50 \mathrm{~mm}$.

A curved sparse annular array (see [18]) with six active ring elements and five inactive rings was used (Figure 5). Focusing was realised by synthetic focusing in receive mode, whereby only the central element was used as a sender. Focusing was realised in such a way that the echo signals of the different ring elements were time-shifted and added up. The time shifts were calculated so that constructive interference was achieved for the signal parts originating from the intended focus position. This was done to compensate the different path lengths from the sound focus to the elements.

a)

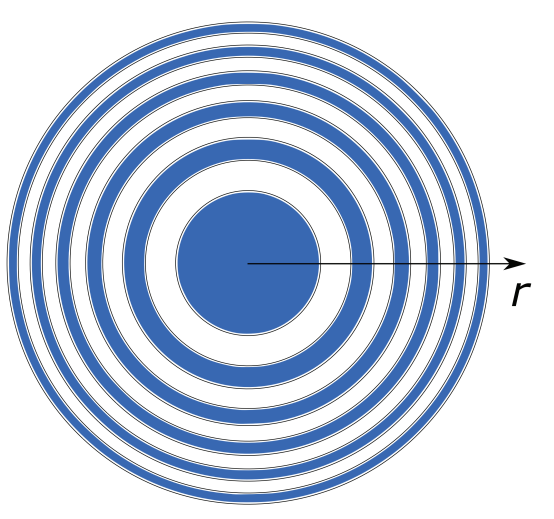

b)

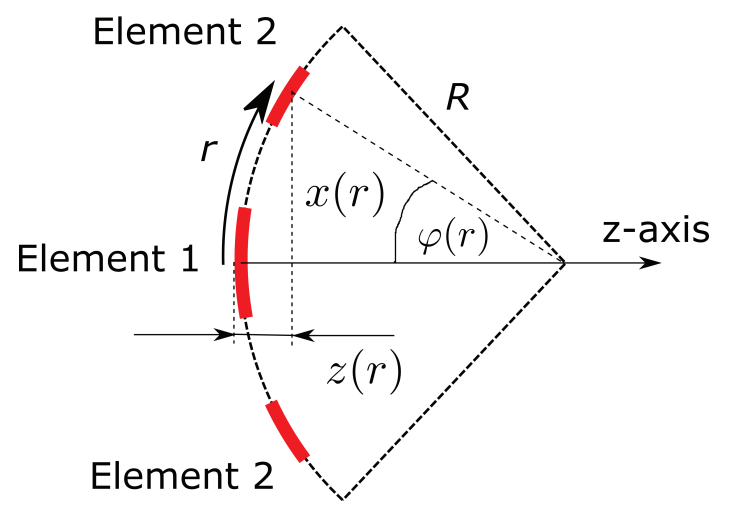

Figure 5 a) Annular array; b) Determination of time shifts.
In the corresponding numerical simulations, the sound fields were calculated by summing up the phase-shifted fields of the transducer segments, where the phase shifts were adjusted on the basis of the nominal geometry of the sparse array.

The results (Figure 6) verify that focusing with an annular array works as well as employing a lens in combination with a plane single element transducer, and that the focus position can truly be varied. Because of the good quality of simulations, we expect feasibility of sound velocity profiles to be confirmed in future measurements.
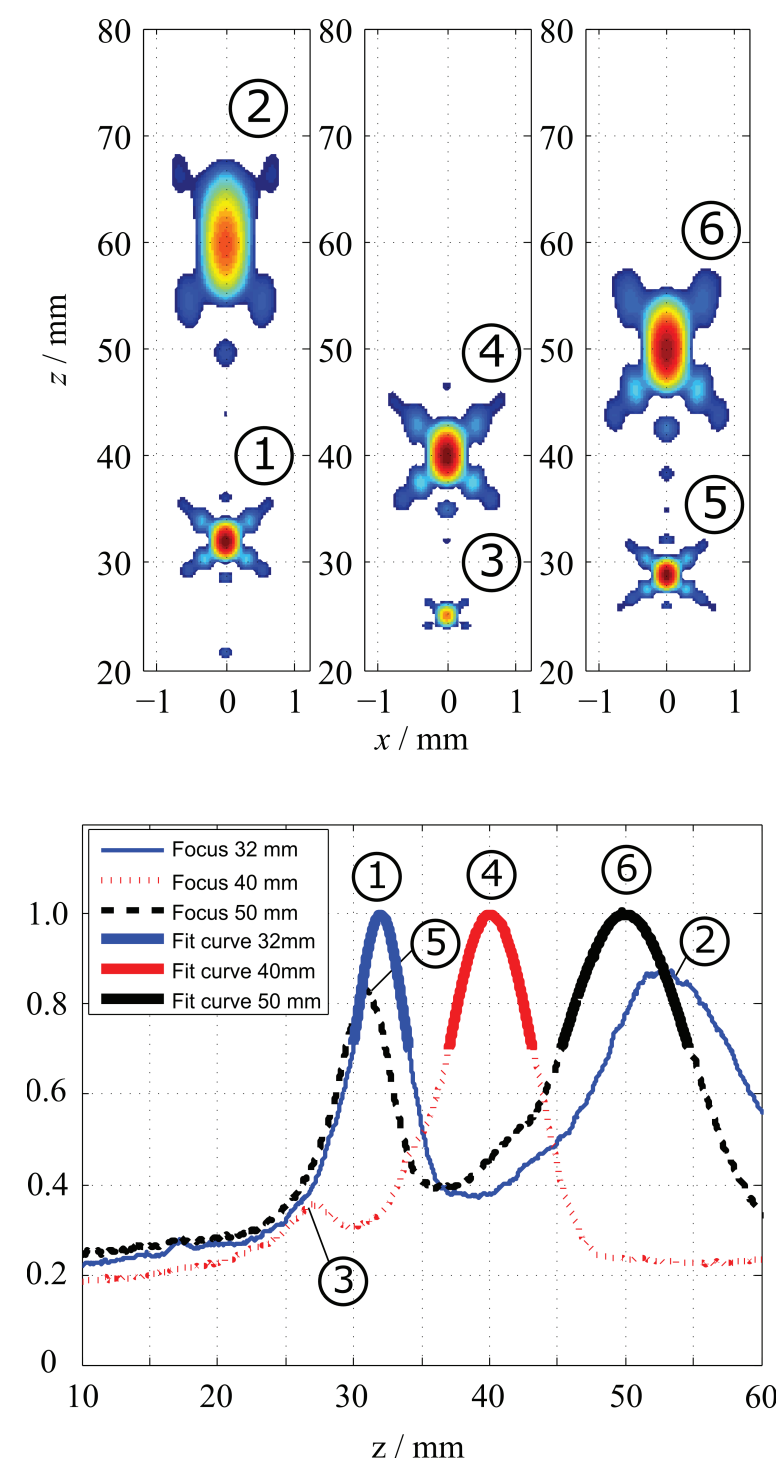

Figure 6 Change of focus position with an annular array. 
Calibration curve for the average sound velocity between transducer and focus position

One possible way to achieve a sound velocity profile is to vary the focus position stepwise and, for each step, determine the average sound velocity between ultrasonic transducer and focus position. The average sound velocity to the focus can then be determined with a parameterised function, one of which being shown as an example in Figure 7. Here, the combination of intended focus position (defined by a set of time-shifts for the different ring elements) and the measured time of flight to the focus yield the average sound velocity to the focus position.

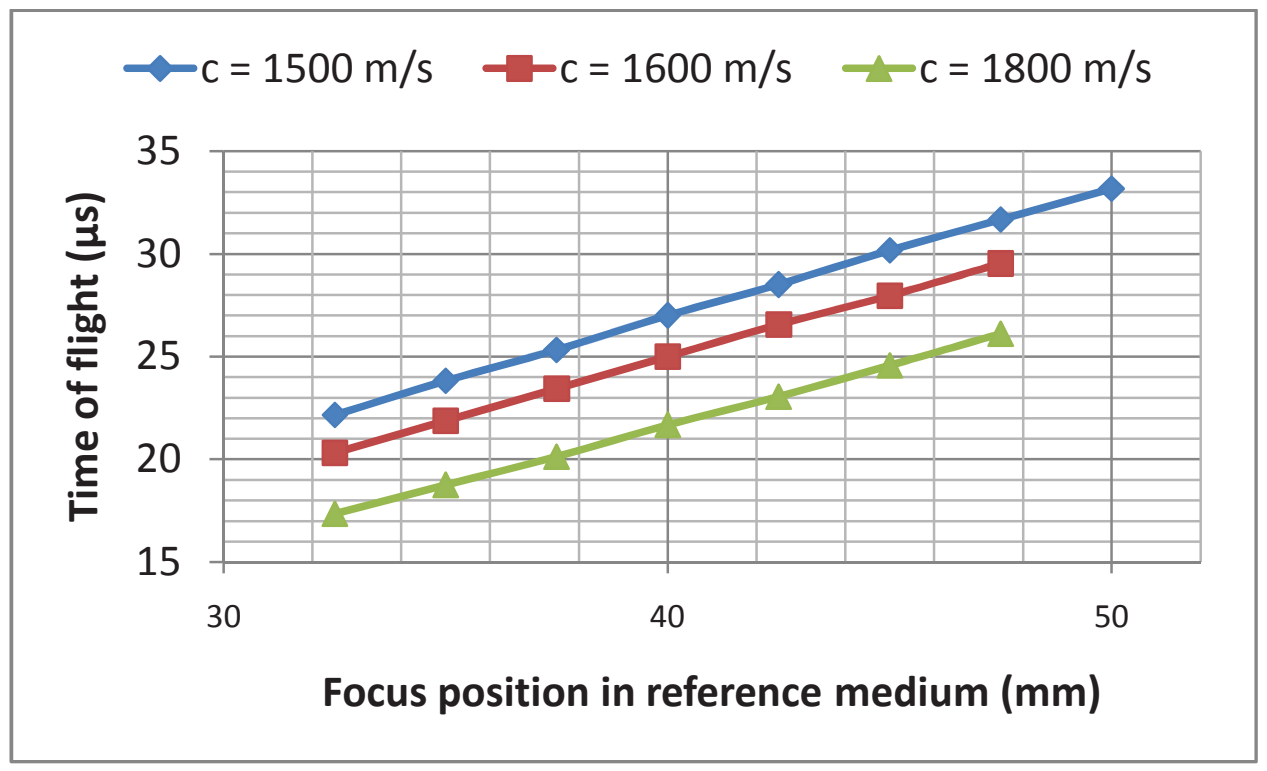

Figure 7 Calibration function for determination of sound velocity profiles.

\section{Conclusion}

A novel approach for non-invasive sound velocity measurement using scattering particles in a fluid is explained and demonstrated by theory and simulations. Good agreement between experiments and the numerical sound field simulations is found. The possibility to change the focus depth with an annular array allows measuring the sound velocity with local resolution.

\section{Acknowledgement}

This research was partially supported by "Bundesministerium für Wirtschaft und Technologie" (Project number: Jülich FKZ 03VWP0022) and "Deutsche Forschungsgemeinschaft" (Project number KU1075/11-1).
This suggests that it is possible to measure velocity profiles along the axis of sound propagation without the need for any transducer movement. For that purpose, a calibration curve showing the average sound velocity between transducer and focus is given.

Corresponding measurements in layered media with differing sound velocities and for fluids with a temperature gradient are planned for the future. 


\section{References}

[1] A. Zips, Prozeßkontrolle mittels Ultraschall, Technisches Messen 67:5 (2000) 201-207. DOI: 10.1524/teme.2000.67.5.201

[2] G. Steiner, C. Deinhammer: Ultrasonic time-of-flight techniques for monitoring multi-component processes, Elektrotechnik und Informationstechnik, Springer, 2009. DOI: 10.1007/s00502-009-0640-6

[3] R. C. Asher: Ultrasonics in chemical analysis, Ultrasonics 25:1 (1987) 17-19. DOI: 10.1016/0041-624X(87)90004-7

[4] N. Hoppe, G. Schönfelder, P. Hauptmann, Ultraschall-Dichtesensor für FlüssigkeitenEigenschaften und Grenzen, Technisches Messen 69:3 (2002) 131-137. DOI: 10.1524/teme.2002.69.3.131

[5] U. Kaatze, F. Eggers, K. Lautscham, Ultrasonic velocity measurements in liquids with high resolution-techniques, selected applications and perspectives. Meas. Sci. Technol. 19 (2008) 062001. DOI: 10.1088/0957-0233/19/6/062001

[6] J. Krautkrämer, H. Krautkrämer: Ultrasonic testing of materials, fourth ed., Springer, Berlin, 1990.

[7] R. Lerch, G. Sessler, D. Wolf, Technische Akustik- Grundlagen und Anwendungen, Springer, Berlin, 2009. DOI: 10.1007/978-3540-49833-9

[8] A. D. Pierce: Acoustics-An introduction to its physical principles and applications, Acoustical Society of America, Melville, NY, 1989. ISBN 0-88318-612-8

[9] E. Kühnicke, Three-dimensional waves in layered media with nonparallel and curved interfaces: A theoretical approach, J. Acoust. Soc. 100:2 (1996) 709-716. DOI: 10.1121/1.416219

[10] E. Kuehnicke, Plane arrays - Fundamental investigations for correct steering by means of sound field calculations, Wave Motion 44 (2007) 248-261. DOI:

10.1016/j.wavemoti.2006.10.003

[11] E. Kühnicke, Elastische Wellen in geschichteten Festkörpersystemen: Modellierungen mit Hilfe von Integraltransformationsmethoden; Simulationsrechnungen für Ultraschallanwendungen, TIMUG e.V., Bonn, 2001.

[12] W. Marczak, Water as a standard in the measurements of speed of sound in liquids, J. Acoust. Soc. Am. 102:5 (1997) 27762779. DOI: $10.1121 / 1.420332$
[13] E. B. Freyer, J. C. Hubbard, D. H. Andrews, Sonic studies of the physical properties of liquids. I. The sonic interferometer. The velocity of sound in some organic liquids and their compressibilities, J. Am. Chem. Soc. 51:3 (1929) 759-770. DOI: 10.1021/ja01378a014

[14] M. Brai, U. Kaatze, Ultrasonic and hypersonic relaxations of monohydric alcohol/water mixtures, J. Phys. Chem. 96:22 (1992) 8946-8955. DOI: $10.1021 / \mathrm{j} 100201 \mathrm{a} 046$

[15] C. J. Burton, A study of ultrasonic velocity and absortpion in liquid mixtures, J. Acoust. Soc. Am. 20:2 (1948) 186-199. DOI: 10.1121/1.1906362

[16] N. Herrmann, D. J. McClements, Influence of viscoinertial effects on the ultrasonic properties of monodis perse silica suspensions, J. Acoust. Soc. Am. 106:2 (1999) 1178-1181. DOI: 10.1121/1.427127

[17] A. Cramer, C. Zhang, S. Eckert, Local flow structures in liquid metals measured by ultrasonic Doppler velocimetry, Flow Meas. Instrum. 15:3 (2004) 145-153. DOI: 10.1016/j.flowmeasinst.2003.12.00

[18] J. A. Brown, G. R. Lockwood, Design of Sparse Annular Arrays for High-Frequency Imaging, Ultrasym. (2005) 125-128. DOI: 10.1109/ULTSYM.2005.1602812

[19] E. Kühnicke, M. Lenz: Anordnung und Verfahren zur kombinierten Bestimmung von Schallgeschwindigkeiten und Abständen in flüssigen und festen Medien mittels Ultraschall. TU PAT 0306092009.

[20] E. Kühnicke, M. Lenz: Anordnung und Verfahren zur Bestimmung von Schichtdicken und Schallgeschwindigkeiten in Medien mit Hilfe von Ultraschall. TU PAT 0406092009.

[21] Elfgard Kühnicke, Michael Lenz, Norbert Gust: Neue Ultraschallmessverfahren, Sensoren und Messsysteme 2010, 18.19.05.2010 Nürnberg, ISBN 978-3-80073260-9, VDE Verlag Berlin-Offenbach, S. 280-286. 